\title{
Ordered and Disordered Defect Chaos
}

\author{
Glen D. Granzow and Hermann Riecke \\ Department of Engineering Sciences and Applied Mathematics \\ Northwestern University, Evanston, IL 60208, USA
}

\begin{abstract}
Defect-chaos is studied numerically in coupled GinzburgLandau equations for parametrically driven waves. The motion of the defects is traced in detail yielding their life-times, annihilation partners, and distances traveled. In a regime in which in the one-dimensional case the chaotic dynamics is due to double phase slips, the two-dimensional system exhibits a strongly ordered stripe pattern. When the parity-breaking instability to traveling waves is approached this order vanishes and the correlation function decays rapidly. In the ordered regime the defects have a typical life-time, whereas in the disordered regime the life-time distribution is exponential. The probability of large defect loops is substantially larger in the disordered regime.
\end{abstract}

$$
\text { April 19, } 1997
$$

Paper for the proceedings of the $\mathrm{V}^{\text {th }}$ Bar-Ilan Conference Physics of Complex Systems.

\section{INTRODUCTION}

Spatio-temporal chaos has been studied in quite some detail experimentally and theoretically. One of the difficulties in studies of such complex states is the identification of quantities that allow a suitable characterization of the complex dynamics and of transitions that might occur between different types of dynamics. A large number of theoretical investigations has focussed on the complex Ginzburg-Landau equation, which describes the dynamics of weakly nonlinear waves. There, spatio-temporal chaos arises over a wide range of parameters. In one dimension two regimes have been identified, one (amplitude chaos) in which the dynamics is dominated by phase slips, i.e. events during which the amplitude of the wave goes to zero and a wavelength is inserted or eliminated, and a regime in which (essentially) no phase slips occur (phase chaos) [1]:2]. In two dimensions the role of the phase slips is played by the creation and annihilation of defects in the wave pattern, which in this system have the form of spirals [3]. A number of different regimes of such spatio-temporal chaos have been identified [4,5].

Since the spirals are topologically stable and easily identified it is natural to attempt a characterization of the chaotic state in terms of the dynamics and statistics of these defects. It has been found that the probability distribution function for the number of defect pairs is consistent with a model in which the defects are created randomly in pairs and annihilated by each other at a rate that is proportional to their density, i.e. they appear to be statistically independent of each other [6]. This was also found in experiments on electro-convection in nematic liquid crystals $[7]$. Since the defects are always created in pairs of opposite 'charge' the total charge is conserved and the charge density is expected to evolve on large space- and time-scales. This was investigated in [8]. There, it was also remarked that the defects are typically not bound in pairs, i.e. they are usually annihilated by a defect that is not the defect they were created with. No detailed analysis of this question has been performed, however.

In this paper two-dimensional spatio-temporal chaos is investigated in a model for parametrically excited waves. The work is motivated by previous results in one dimension, which identified a regime in which the dynamics is due to double phase slips rather than single phase slips [9,10]. Double phase slips consist of a sequential pair of phase slips in which the second phase slip quickly follows and negates the first. Across a double phase slip the total phase of the system is conserved. Thus, the waves have a well-defined effective wave number and a large-scale description of the chaotic state with an effective phase diffusion equation is possible [9, 10]. In two dimensions the double phase slips are expected to correspond to 'fluctuating bound defect pairs', i.e. to pairs of defects that are created together and are annihilated by each other after a typical time during which the defects remain strongly correlated. Preliminary computations have indicated such a behavior [10]. An interesting question concerns the possibility of an 'unbinding' of these defect pairs and as a consequence of a transition from a more ordered to a less ordered chaotic state. Here we present computations which show a drastic decrease in the order of the chaotic state connected with the disappearance of the typical lifetime of the defects and other signatures in the defect statistics.

\section{THE MODEL EQUATIONS}

In this paper we study the dynamics of parametrically excited waves within the framework of coupled GinzburgLandau equations. These describe the temporal evolution of the complex amplitudes $A$ and $B$ of oppositely traveling waves that are coupled due to a parametric forcing at a frequency $\omega_{e}$, which is close to twice the frequency of the traveling waves. The traveling waves are assumed to arise in a supercritical Hopf bifurcation. In terms of the amplitudes $A$ and $B$, physical quantities $u$ like the vertical fluid velocity in the mid-plane of a convection system are given by 


$$
\begin{array}{r}
u(\mathbf{r}, t)=\epsilon A(\mathbf{R}, T) e^{i\left(\mathbf{q}_{c} \cdot \mathbf{r}-\frac{\omega_{e}}{2} t\right)}+\epsilon B(\mathbf{R}, T) e^{i\left(\mathbf{q}_{c} \cdot \mathbf{r}+\frac{\omega_{e}}{2} t\right)} \\
+ \text { c.c. }+o(\epsilon) .
\end{array}
$$

The amplitudes $A$ and $B$ vary on the slow time and space scales, $T=\epsilon^{2} t, \mathbf{R} \equiv(X, Y)=\epsilon \mathbf{r}$ with $\mathbf{r}=(x, y)$ and $\epsilon \ll 1$.

The ansatz (11) introduces an anisotropy into the system since the slowly varying coordinate $\mathbf{R}$ does not capture large variations in the orientation of the waves. This leads to difficulties in the description of isotropic physical systems. For their description one is therefore either forced to restrict oneself to situations in which the orientation of the wavenumber varies only slightly or one has to allow the amplitudes to vary on the fast scale r. In the former case a systematic derivation leads to coupled Newell-Whitehead-Segel equations for the amplitudes (e.g. 11]). In the latter case one obtains a complex order-parameter equation in which the nonlinearities contain complicated integral terms (e.g. [12]). Usually, these are then approximated by local terms (e.g. 12,13]).

If the physical system has an axial anisotropy no such difficulties arise since the waves travel essentially along the preferred direction as is, for instance, the case in electro-convection in nematic liquid crystals [7, 14, 15]. In that case, if the waves arise in a supercritical bifurcation, they can be described by coupled Ginzburg-Landau equations of the form 16, 17

$$
\begin{gathered}
\partial_{T} A+s \partial_{X} A=d \nabla^{2} A+a A+b B \\
+c A\left(|A|^{2}+|B|^{2}\right)+g A|B|^{2}, \\
\partial_{T} B-s \partial_{X} B=d^{*} \nabla^{2} B+a^{*} B+b A \\
+c^{*} B\left(|A|^{2}+|B|^{2}\right)+g^{*} B|A|^{2} .
\end{gathered}
$$

The coefficients in (2/3) are complex except for $s$ and $b$, which are real. The real part $a_{r}$ of the coefficient $a$ gives the linear damping of the traveling waves in the absence of the periodic forcing and is proportional to the distance from the Hopf bifurcation. The coefficient of the linear coupling term $b$ gives the amplitude of the periodic forcing as can be seen from the fact that it breaks the continuous time-translation symmetry $t \rightarrow t+\Delta t$, which implies the transformation $A \rightarrow A e^{i \Delta t \omega_{e} / 2} B \rightarrow$ $B e^{-i \Delta t \omega_{e} / 2}$. The imaginary part $a_{i}$ of the coefficient $a$ gives the difference between the frequency of the unforced waves (at wavenumber $\mathbf{q}_{c}$ ) and half the forcing frequency $\omega_{e}$.

In addition to the trivial solution $A=B=0$, (2,3) possess three types of simple solutions: $|A|=|B|=$ const, $|A| \neq|B|$ (both constant), and $|A|=|B|$ (both timeperiodic) [16]. We are in particular interested in the first type, which corresponds in the physical system to standing waves that are phase-locked to the parametric forcing, i.e. they are excited by the forcing. With increasing $a_{r}$ they become unstable to solutions of the second type, which correspond to traveling waves as they exist also in the absence of any periodic forcing. Solutions of the third type correspond to standing waves that are not phase-locked to the forcing. For the case discussed here, $g_{r}<0$, they are unstable to the traveling waves.

The stability of the phase-locked standing waves with respect to long-wave modulations (Eckhaus instability) can be described using a phase-diffusion equation. The stability limits are then given by a sign-change of the diffusion coefficient. For the one-dimensional case the diffusion coefficient was determined analytically in [18]. The phase-diffusion equations does not describe the paritybreaking instability, at which the standing waves become unstable to traveling waves 16]. Fig.11 shows the stability limits relevant for the present paper. The neutral curve, above which the trivial state is unstable to standing waves, is given by the dashed line. Their Eckhaus instability is denoted by a solid line and their parity-breaking instability by a dashed-dotted line. Over some range of parameters the parity-breaking instability is preempted by a mode that arises first at finite modulation wavenumber (open squares). It emerges from the parity-breaking instability. The standing waves are stable only inside the region marked by the solid lines and the squares. For completeness it should be noted that to the left of the minimum of the neutral curve the bifurcation to standing waves becomes subcritical; the saddle-node line is shown as a thin long-dashed line. For negative $Q$ a second minimum of the neutral curve exists, but the only stable regime of the standing waves is as shown in fig.1. Traveling waves exist below the parity-breaking instability and to the left of the neutral curve indicated by the dotted line at $Q=0.5$.

As in the case studied in 18, 10, 10, in which $a_{r}<0$ and no traveling waves arise, the region of stable standing waves is closed in fig.1] $\left(a_{r}=0.25\right)$ with the standing waves becoming Eckhaus-unstable for large forcing. In one dimension, it was found that for larger $b$ the Eckhaus instability does not lead to a single phase slip, which would change the overall wavenumber of the standing waves and would thus take the unstable waves into the stable band, but instead to double phase slips. They consist of a sequential pair of phase slips with the second phase slip undoing the action of the first one. Therefore the total phase is conserved across such a double phase slip and the overall wavenumber remains the same. As a consequence, persistent dynamics - regular and chaotic - can arise. In particular, it was found that the chaotic dynamics can become localized in space, a phenomenon that can be understood within the framework of an effective phase diffusion equation [9,10].

In the complex Ginzburg-Landau equation describing unforced traveling waves no regime with double phase slips seems to exist. With increasing $a_{r}$ the parametrically excited standing waves become unstable to traveling waves in a parity-breaking instability [16,14]. The latter are only weakly affected by the forcing for sufficiently large $a_{r}$. One may therefore expect an interesting transition from chaotic dynamics characterized by double phase slips to one dominated by single phase slips when $a_{r}$ is increased towards the parity-breaking instability. 
In two dimensions the overall wavenumber is changed by dislocations. A single phase slip is effected by a pair of dislocations being created and separating to infinity, i.e. disappearing at opposite walls of the system. Since the two phase slips in a double phase slip undo each other one might expect that the double phase slips correspond to a process in which two dislocations are created and move apart, but then turn around and annihilate each other again. Thus, they would appear as if they were bound to each other. Preliminary calculations have shown this behavior 10.

\section{ORDERED AND DISORDERED CHAOTIC WAVES}

To investigate the dynamics of defects in detail we simulate (2,3) numerically using a pseudo-spectral method with a $4^{t h}$-order Runge-Kutta/integrating factor timestepping scheme. The time step is $d t=0.25$ and the highest Fourier mode is $N=64$ for a system length $L=136$. Periodic boundary conditions are used. The parameters are chosen as in fig.1. Since $a_{r}>0$, the standing waves become unstable to traveling waves when the forcing $b$ is decreased. It is expected that this parity-breaking instability will also affect the dynamics of the defects, in particular since in 1 dimension the traveling waves do not exhibit any double phase slips. Here we present results for $b=1$, i.e. far away from the parity-breaking instability, and for $b=0.5$, which is much closer to that instability. A typical snapshot of the waves is shown in fig. 2 for $b=1$ and in fig. 3 for $b=0.5$.

For $b=1$ the wave pattern consists of a quite ordered array of stripes with a number of defects. The correlation function in fig. A, which is an average over $\Delta T=20000$ shows that in this regime the correlation function decays only very little over the whole system. In analogy to the one-dimensional chaotic state that is driven by double phase slips, it is expected that the large-scale dynamics of this stripe pattern can be described by a diffusion equation with noise $[10$. No true long-range order is expected in such a stripe pattern. For the system sizes investigated so far $(L=68$ and $L=136)$, we could not address this question. Since the pattern has a quite well-defined wavenumber the dynamics depends somewhat sensitively on the length, which discretizes the possible wavenumbers. When the effective wavenumber is decreased (by increasing the length of the system) the pattern develops into a zig-zag pattern, very similar to what is observed in non-chaotic systems. To avoid this complication we have adjusted the length such that no zig-zags were apparent.

For $b=0.5$ the wave pattern is quite disordered. The correlation function in fig.5 (averaged over $\Delta T=$ 10000) shows that the pattern becomes statistically almost isotropic in this case (despite the small anisotropy due to the group velocity $s=0.2$ ). The decay of the correlation function is clearly exponential.
To characterize the dynamics of the defects they are tracked from their creation to their annihilation. In order to address the question whether there are 'bound' defect pairs each defect pair is labeled and it is noted which defect is annihilated by which other defect. In space-time the defects trace out loops 10. If a defect is annihilated by the same defect with which it was created such a loop consists only of this defect pair and is opened when they are created and closed when they annihilate each other. In general, the loops can consist of any number of defect pairs.

In fig.6 the probability distribution function for the number of defect pairs making up a loop is shown for $b=1$ and $b=0.5$. In both cases most loops contain only a single defect pair. At first glance this might suggest that the defects are bound to each other in both cases. This is, however, not the case as the other diagnostics indicate. In fact, even in the single Ginzburg-Landau equation, in which no double phase slips occur in 1 dimension and for which no bound defects are expected, most loops contain only a single defect pair 19]. A strong difference between the two regimes arises, however, in the probability of multi-loops: loops containing many defects are much more likely for $b=0.5$ than for $b=1$. In fact, from the data available so far it is not clear whether the probability decays exponentially for $b=0.5$.

Another striking difference in the two regimes is noticed in the distribution of the life-times of each defect. As seen in fig.7 the distribution has a clear peak for $b=1$ indicating a typical life-time of the individual defects while for $b=0.5$ the distribution is exponential over the whole range. The latter indicates that during each time interval a defect has a certain probability to be annihilated; correlations between defects appear not to be important.

An interesting quantity is the 'size' of the loops, i.e. the distance in the $X$ - and in the $Y$-direction that is covered by a loop during its life. To determine this size it has to be taken into account that, due to the periodic boundary conditions, loops can 'wrap around' the system and can, in principle, become larger than the system size. The results are shown in fig. 8. Again, there is a strong difference between the two parameter regimes. While for $b=0.5$ there is a substantial number of loops that cover a large part of the system in the $Y$ - as well as the $X$-direction, the loops remain very restricted in the $X$ direction in the ordered regime, $b=1$. Thus, for $b=1$ the defects exhibit essentially only climb-motion while for $b=0.5$ they travel in all directions. The latter changes the orientation of the pattern and is responsible for the near-isotropy in the correlation function.

\section{CONCLUSION}

In this paper we have presented the results of numerical simulations of a model for parametrically driven waves in 
a two-dimensional anisotropic system. Two types of behavior were found: in a regime in which in 1 dimension all phase slips occur in sequential pairs the two-dimensional waves form an ordered stripe pattern with a very slowly decaying correlation function; for smaller forcing, i.e. closer to the parity-breaking instability, the waves form a very disordered pattern with a rapidly decaying correlation function which is almost isotropic. An analysis of various statistics of the motion of the defects involved in the dynamics shows that in the ordered regime they have a typical life-time whereas in the disordered regime the distribution function is purely exponential. The number of defect pairs in the space-time loops traced out by the defects increases strongly in the disordered regime. Another signature of the disordered state is the extension of the loops. In the disordered regime they extend over large portions of the system in the $X$ - and the $Y$ direction, while in the ordered regime their extension in the $X$-direction is strongly restricted.

At the present it is not clear whether there is a true transition separating the two regimes or whether the change from one to the other is smooth. This needs to be investigated. The extension in the $X$-direction should be a good diagnostic tool for this question.

An interesting question is whether there is any connection between the disappearance of spatial order in this spatio-temporally chaotic system and two-dimensional melting [20 and the Kosterlitz-Thouless transition 21] in equilibrium systems.

HR gratefully acknowledges discussions with L. Kadanoff, R. Lipowsky and J. Marko. This work was supported by DOE through grant DE-FG02-92ER14303 and made use of the resources of the Cornell Theory Center, which receives major funding from NSF and New York State with additional support from the Advanced Research Projects Agency, the National Center for Research Resources at the National Institutes of Health, IBM Corporation and members of the Corporate Research Institute.

[1] B.I. Shraiman, A. Pumir, W. van Saarloos, P.C. Hohenberg, H. Chaté, and M. Hofen. Spatiotemporal chaos in the one-dimensional complex Ginzburg-Landau equation. Physica D, 57:241, 1992.

[2] D.A. Egolf and H.S. Greenside. Characterization of the transition from defect to phase turbulence. Phys. Rev. Lett., 74:1751-1754, 1995.

[3] P. Coullet and J. Lega. Defect-mediated turbulence in wave patterns. Europhys. Lett., 7:511, 1988.

[4] I. Aranson, L. Kramer, and A. Weber. Core instability and spatiotemporal intermittency of spiral waves in oscillatory media. Phys. Rev. Lett., 72:2316, 1994.

[5] H. Chaté and P. Manneville. Phase diagram of the two- dimensional complex Ginzburg-Landau equation. Physica A, 224:348, 1996.

[6] L. Gil, J. Lega, and J.L. Meunier. Statistical properties of defect-mediated turbulence. Phys. Rev. A, 41:1138-1141, 1990.

[7] I. Rehberg, S. Rasenat, and V. Steinberg. Traveling waves and defect-initiated turbulence in electroconvecting nematics. Phys. Rev. Lett., 62:756, 1989.

[8] B.W. Roberts, E. Bodenschatz, and J.P. Sethna. A bound on the decay of defect-defect correlation functions in two-dimensional complex order parameter equations. Physica D, 99:252, 1996.

[9] G.D. Granzow and H. Riecke. Phase diffusion in localized spatio-temporal amplitude chaos. Phys. Rev. Lett., 77:2451, 1996.

[10] G.D. Granzow and H. Riecke. Double phase slips and spatio-temporal chaos

in a model for parametrically excited standing waves. SIAM J. Appl. Math., submitted.

[11] M.C. Cross and P.C. Hohenberg. Pattern formation outside of equilibrium. Rev. Mod. Phys., 65:851, 1993.

[12] M. Neufeld, R. Friedrich, and H. Haken. Order-parameter equation and model equation for high Prandtl number Rayleigh-Bénard convection in a rotating large aspect ratio system. Z. Phys. B, 92:243, 1993.

[13] W. Zhang and J. Vinals. Secondary instabilities and spatiotemporal chaos in parametric surface waves. Phys. Rev. Lett., 74:690, 1995.

[14] I. Rehberg, S. Rasenat, J. Fineberg, M. de la TorreJuarez, and V. Steinberg. Temporal modulation of traveling waves. Phys. Rev. Lett., 61:2449, 1988.

[15] M. Dennin, M. Treiber, L. Kramer, G. Ahlers, and D.S. Cannell. Origin of traveling rolls in electroconvection of nematic liquid-crystals. Phys. Rev. Lett., 76:319, 1996.

[16] H. Riecke, J.D. Crawford, and E. Knobloch. Timemodulated oscillatory convection. Phys. Rev. Lett., 61:1942, 1988.

[17] D. Walgraef. External forcing of spatio-temporal patterns. Europhys. Lett., 7:485, 1988.

[18] H. Riecke. Stable wave-number kinks in parametrically excited standing waves. Europhys. Lett., 11:213, 1990.

[19] G.D. Granzow and H. Riecke. unpublished.

[20] D.R. Nelson. Defect-mediated phase transitions, volume 7 of Phase Transitions and Critical Phenomena, chapter 1. Academic, New York, 1983.

[21] J.M. Kosterlitz and D.J. Thouless. Ordering, metastability and phase transitions in two-dimensional systems. $J$. Phys. C, 6:1181, 1973. 


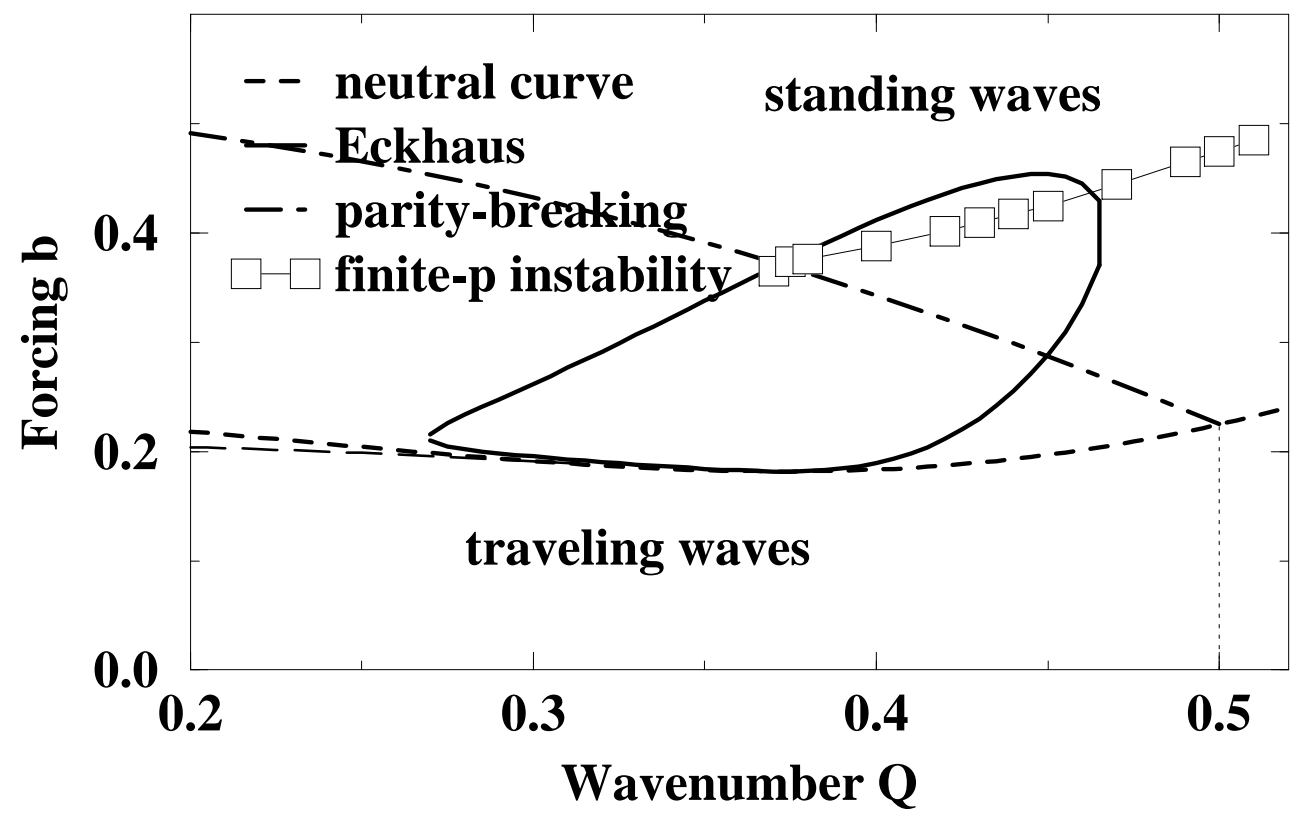

FIG. 1. Linear stability diagram for $a=0.25, c=-1+4 i$, $d=1+0.5 i, s=0.2, g=-1-12 i$. For details see text.

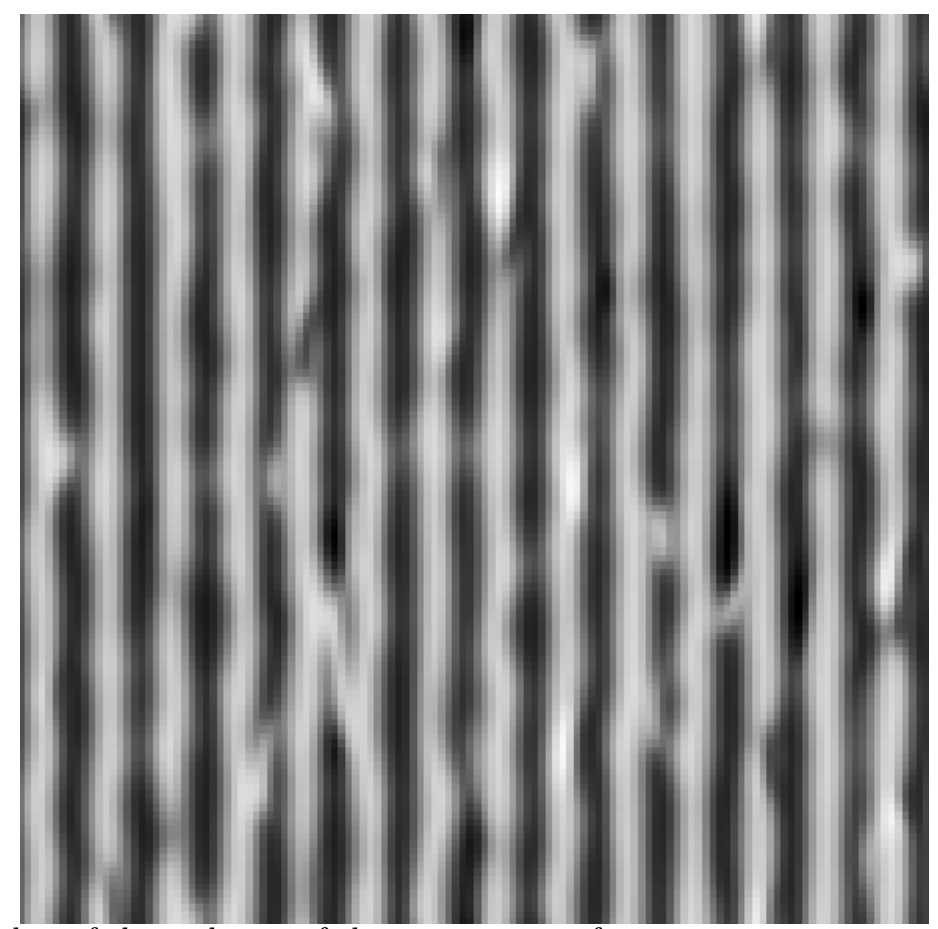

FIG. 2. Snapshot of the real part of the wave pattern for $b=1$ (other parameters as in fig.1). 


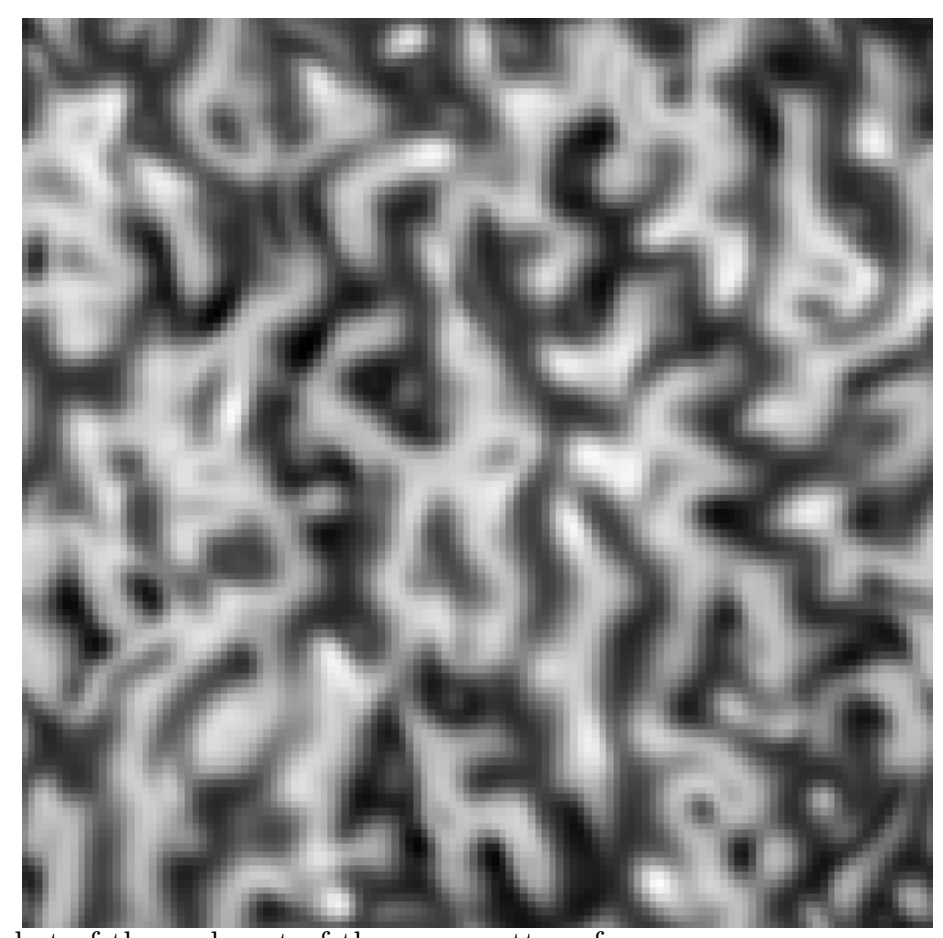

FIG. 3. Snapshot of the real part of the wave pattern for $b=0.5$ (other parameters as in fig.1).

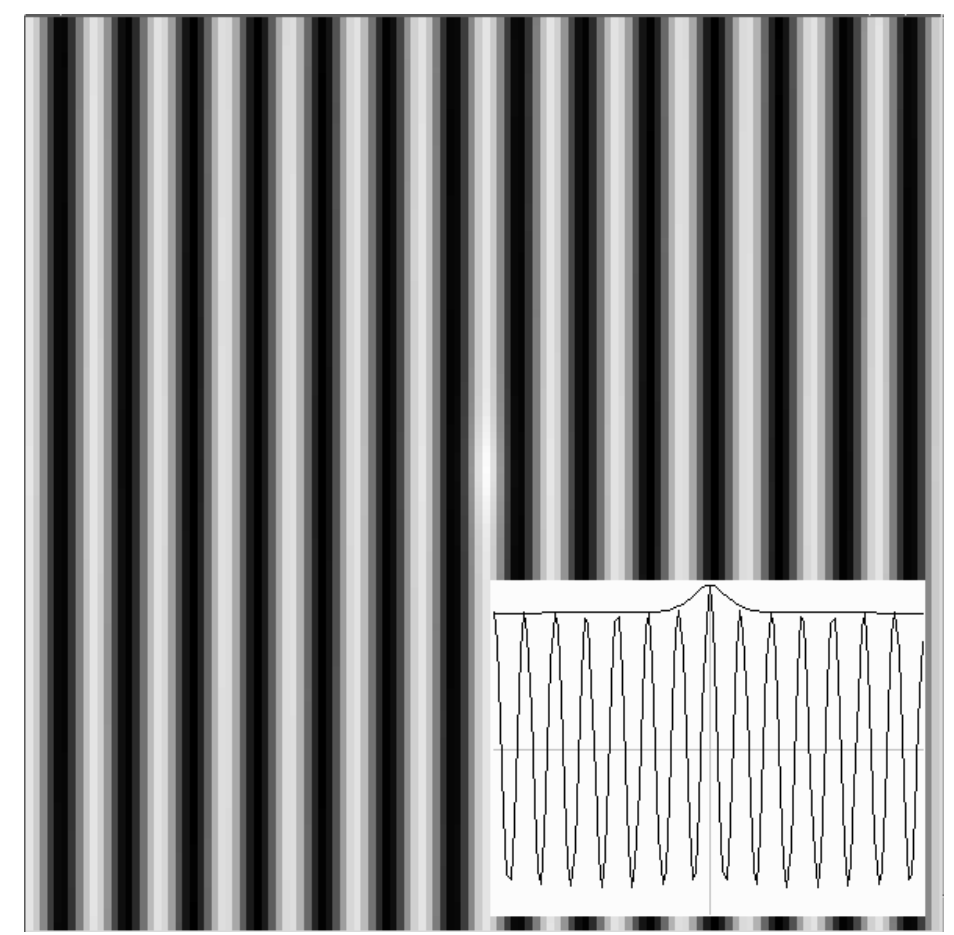

FIG. 4. Time-averaged correlation function of the wave pattern for $b=1$. The inset shows one-dimensional cuts in the $X$ - and $Y$-direction on a linear scale. The other parameters are as in fig.1. 


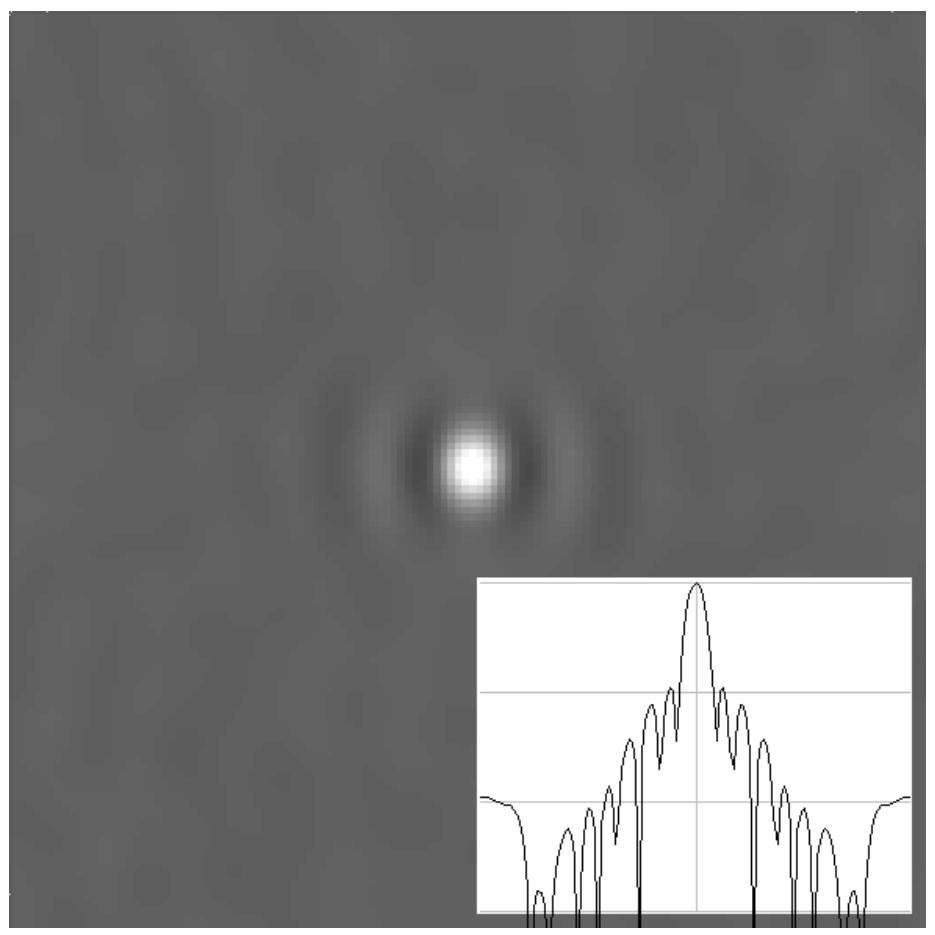

FIG. 5. Time-averaged correlation function of the wave pattern for $b=0.5$. The inset shows a one-dimensional cut in the $X$-direction of the absolute value of the correlation function on a linear-logarithmic scale. The other parameters are as in fig.1.

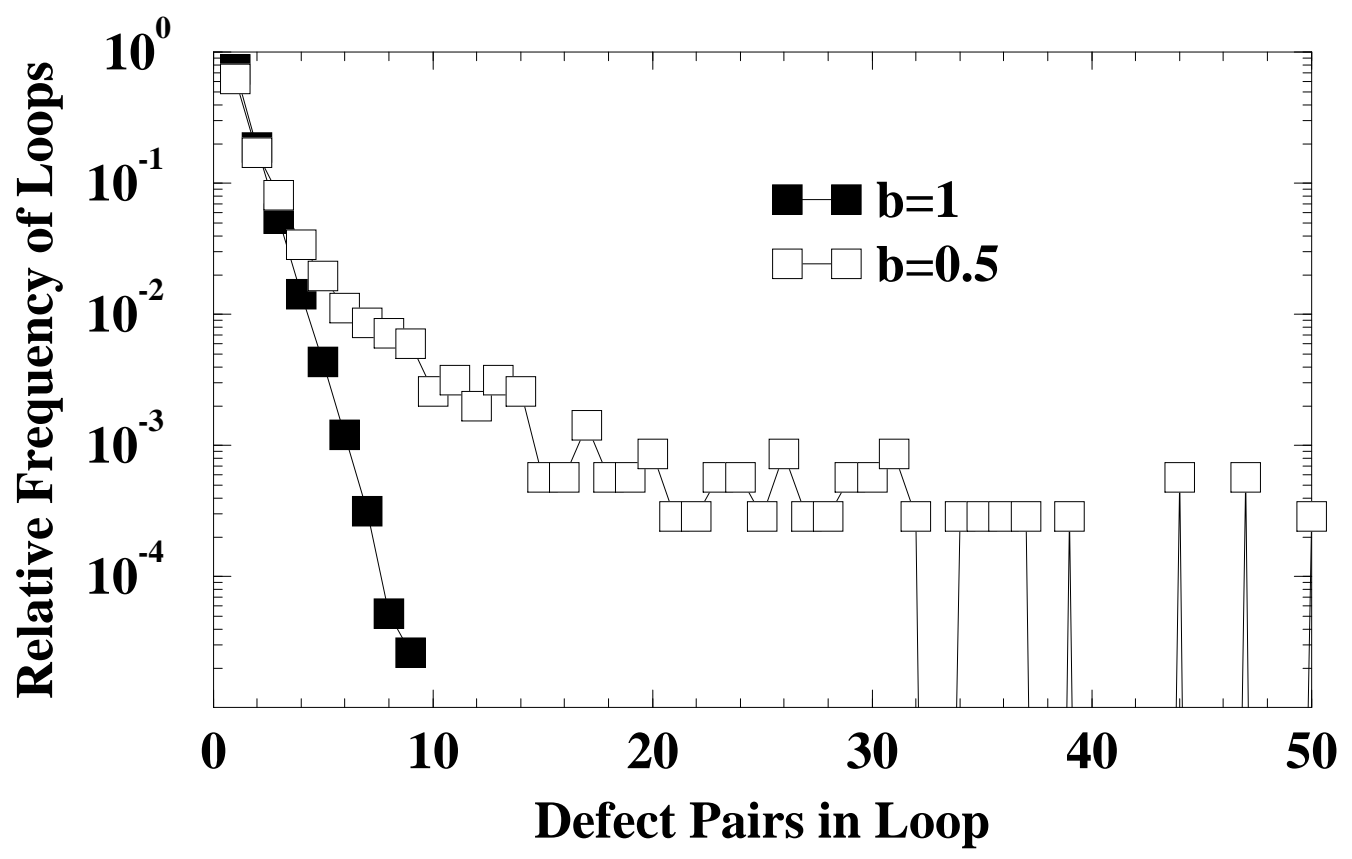

FIG. 6. Relative frequency of defect loops in space-time containing $n$ defect pairs for $b=1$ (solid squares) and $b=0.5$ (open squares). The other parameters are as in fig.1. 


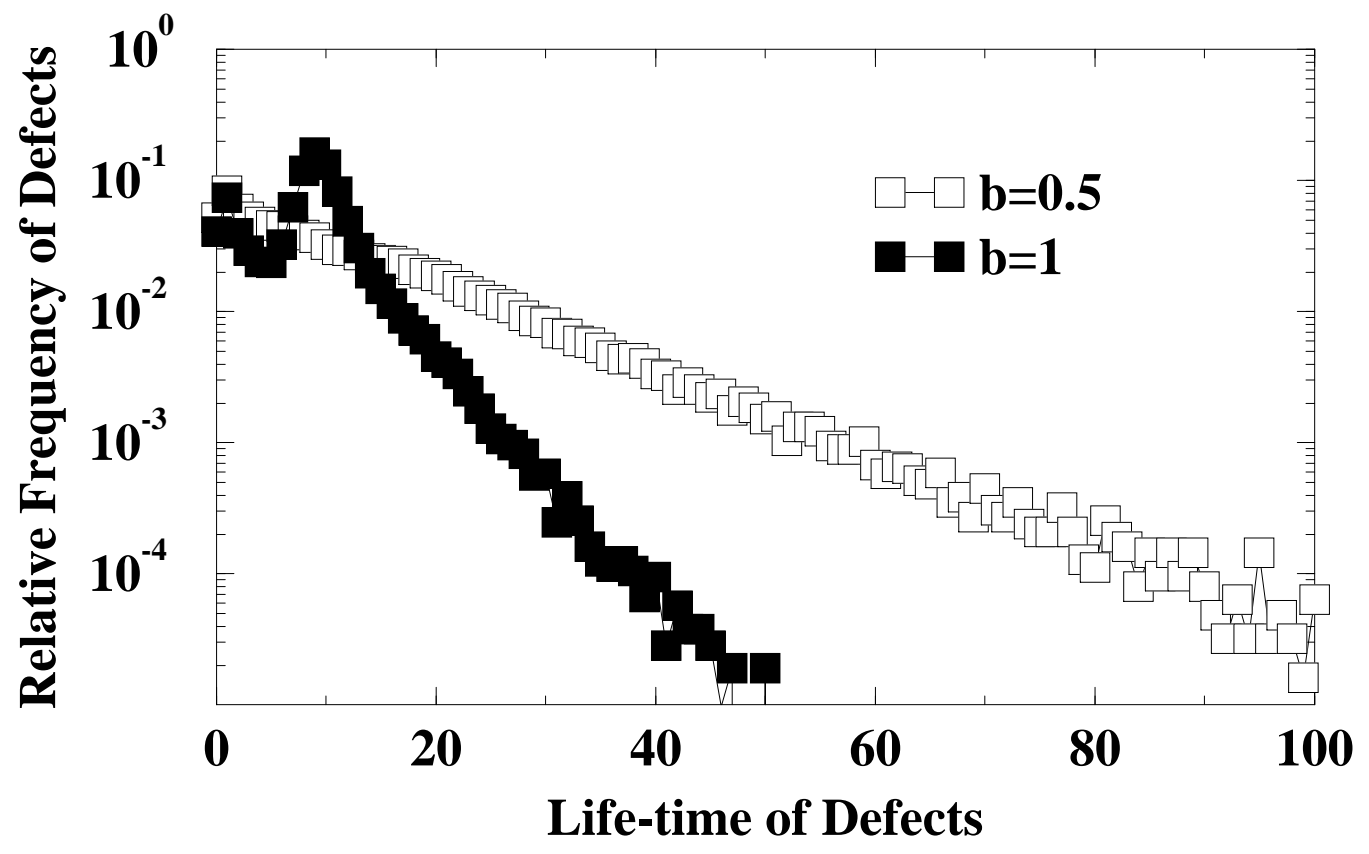

FIG. 7. Relative frequency of defect loops in space-time with life-time $\tau$ for $b=1$ (solid squares) and $b=0.5$ (open squares). The other parameters are as in fig.1.

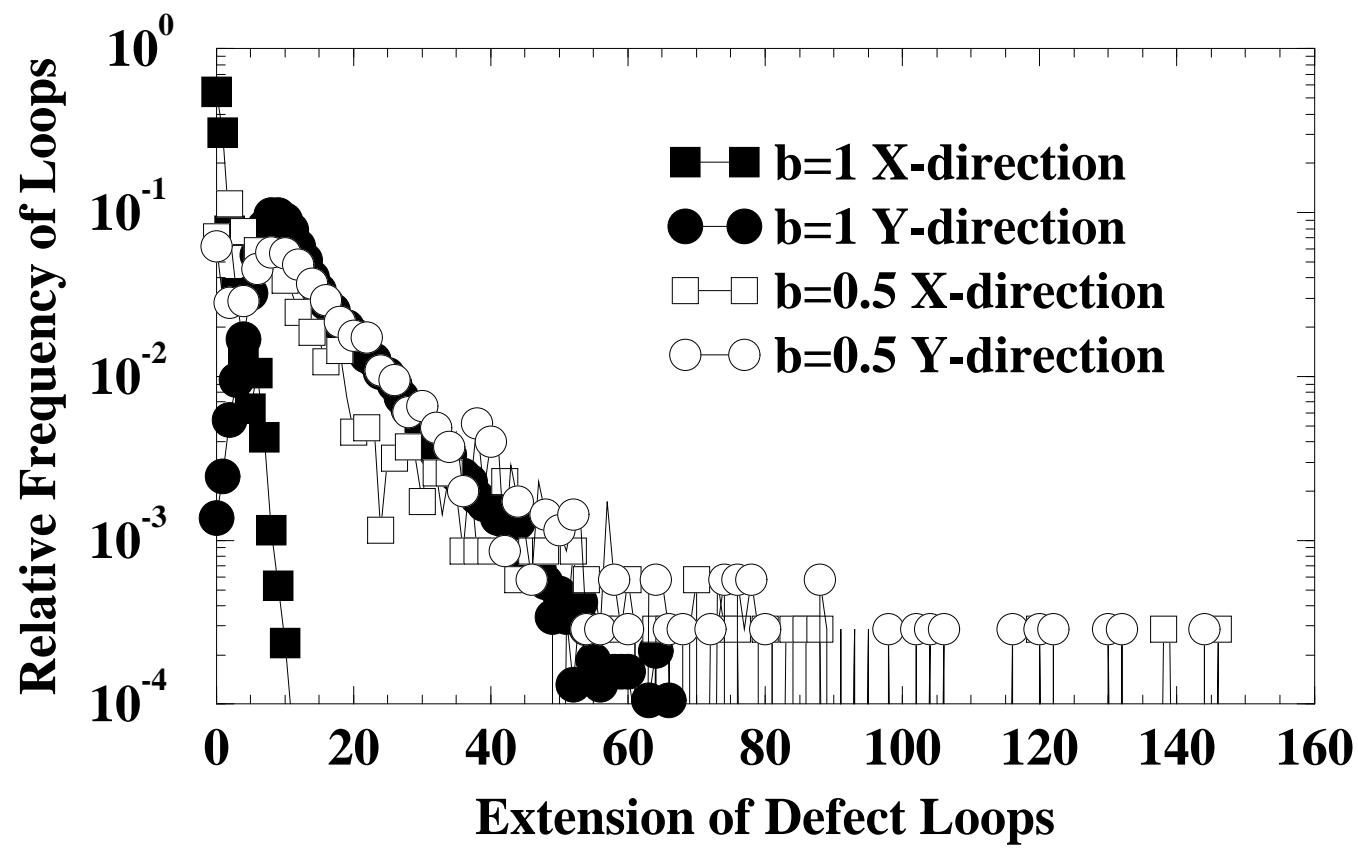

FIG. 8. Relative frequency of defect loops in space-time with extension $d_{x}$ in the $x$-direction (squares) and $d_{y}$ in the $y$-direction (circles) for $b=1$ (solid symbols) and $b=0.5$ (open symbols). The other parameters are as in fig.1. 